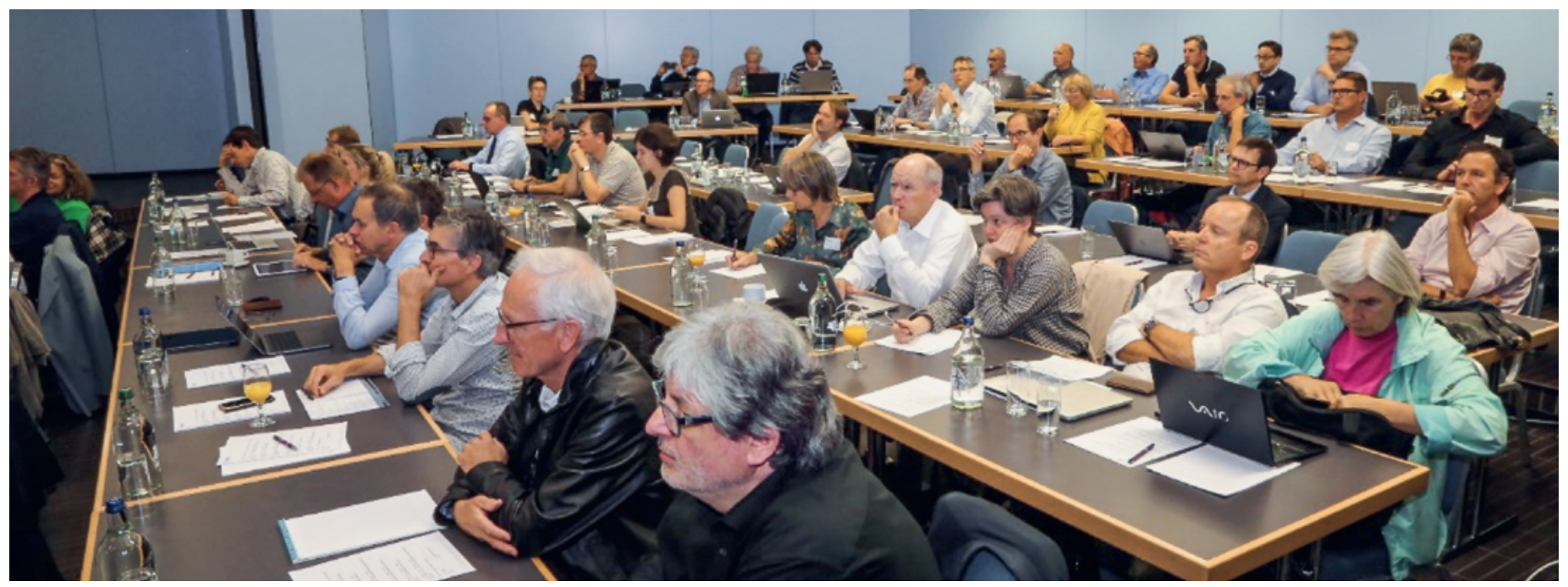

Die Referate stossen bei den Teilnehmern des Tarifdelegierten-Tages auf grosses Interesse.

\title{
Tarifrevision auf der Zielgeraden?
}

\section{Patrick Müller ${ }^{a}$, Thomas Kessler ${ }^{b}$, Susanne Christenc ${ }^{c}$, Christian Oeschger ${ }^{d}$}

${ }^{a}$ FMH, Abteilungsleiter, Abteilung Ambulante Versorgung und Tarife; ${ }^{b}$ FMH, Experte, Abteilung Ambulante Versorgung und Tarife; ${ }^{c}$ Dr. med., FMH, Expertin, Stv. Abteilungsleiterin, Abteilung Ambulante Versorgung und Tarife; ${ }^{d} \mathrm{FMH}$, Experte, Abteilung Ambulante Versorgung und Tarife

Am 27. September 2018 fand in Bern unter dem Titel «Tarifrevision auf der Zielgeraden?» der zweite Tarifdelegierten-Tag dieses Jahres statt. An diesem Tarifdelegierten-Tag nahmen wiederum über 100 Teilnehmer teil. Die Teilnehmerzahl stabilisiert sich auf diesem hohen Niveau.

\section{Politische Einordnung des Tarifvorschlages}

Dr. med. Jürg Schlup, Präsident der FMH, referiert zur politischen Einordnung des vorliegenden Tarifvorschlages. Jürg Schlup beurteilt den TARMED als veraltet und sieht aufgrund des bereits zweimaligen Eingreifens des Bundesrates in den TARMED eine stetige Entwicklung zum Amtstarif. In der Presse und in der Politik sind die Gesundheitskosten ein Dauerthema und es stellt sich die Frage, ob man politische Preise oder einen sachgerechten und betriebswirtschaftlichen Tarif anstreben will. Der Bundesrat will neu in seinem soeben in die Vernehmlassung gegangenen Massnahmenpaket zur Dämpfung der Gesundheitskosten eine Erhöhung der OKP-Kosten gesetzlich korrigieren; diese Korrektur «hat über die Anpassung der Tarife zu erfolgen», und zwar jährlich. Zudem will der Bundesrat eine nationale Organisation zur Pflege der

ambulanten Tarife gesetzlich verankern und falls sich die Tarifpartner nicht auf eine solche nationale ambulante Tariforganisation einigen können, diese selber einsetzen. Deshalb muss die Ärzteschaft jetzt einen

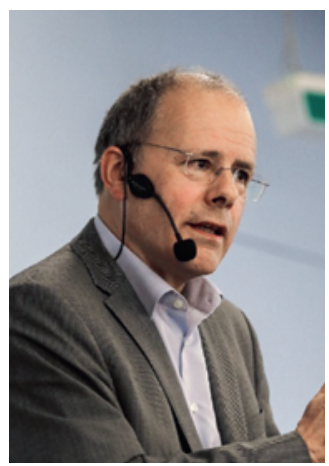

Dr. med. Jürg Schlup referiert über die politische Einordnung des Tarifvorschlages. Vorschlag für eine revidierte Tarifstruktur einbringen, da sonst die Tarifautonomie endgültig verloren geht. Jürg Schlup zieht das Fazit, dass die FMH liefern kann! Dazu haben alle Beteiligten in jahrelanger intensiver Arbeit eine Leistungsstruktur erarbeitet und verhandelt, auf die man stolz sein kann! 


\section{Tarifrevision auf der Zielgeraden?}

Dr. med. Urs Stoffel, Mitglied des Zentralvorstandes der FMH, informiert, dass die Delegiertenversammlung der FMH bzw. die Ärztekammer der FMH die Leistungsstruktur, die im Rahmen des Projektes TARCO erarbeitet wurde, am 2.5.2018 einstimmig verabschiedet hat. Zuvor haben während rund eines Jahres 40 Arbeitsgruppen mit

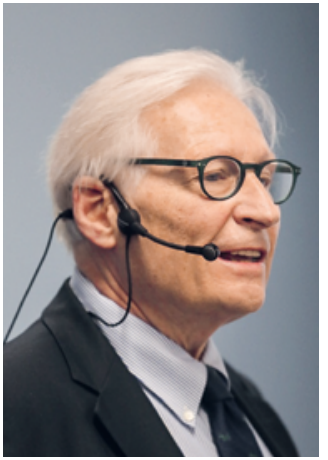

Dr. med. Urs Stoffel referiert darüber, ob die Tarifrevision auf der Zielgeraden ist. mehr als 150 Personen alle Kapitel der Tarifstruktur im Rahmen des Projektes TARCO revidiert. Das Ergebnis aus dem Projekt TARCO floss vollumfänglich in die Verhandlungen mit den Tarifpartnern innerhalb der ats-tms AG ein. Diese Verhandlungen fokussierten sich in den letzten Monaten auf die Integration der Ergebnisse des Projekts TARCO in die neue gemeinsame Leistungsstruktur sowie weiterer Themen. Die Arbeiten an der verhandelten Leistungsstruktur konnten Ende September 2018 abgeschlossen werden, und diese Leistungsstruktur kann nun den FMH-Gremien in diesem Herbst als Etappenziel zur Beschlussfassung vorgelegt werden.

\section{Information zur Vernehmlassung Massnahmenpaket zur Kostendämpfung des Bundes}

Patrick Müller informiert über die laufende Vernehmlassung zu den kostendämpfenden Massnahmen des Bundesrates. Das Eidgenössische Departement des Innern EDI hat Ende 2016 eine internationale Expertengruppe einberufen mit dem Auftrag, rasch umsetzbare kostendämpfende Massnahmen für das Gesundheitswesen vorzuschlagen. Diese Massnahmen sollen gemäss Beschluss des Bundesrates in zwei Paketen umgesetzt werden, wovon das erste seit Mitte September 2018 in der Vernehmlassung ist. Patrick Müller informiert über einzelne Details der Massnahmen. Der Bundesrat möchte, analog wie im stationären Bereich mit SwissDRG AG, eine nationale ambulante Tariforganisation gesetzlich festlegen (Art. 47a KVG neu). Dabei kann der Bundesrat Grundsätze betreffend Form und Betrieb der Organisation auf Verordnungsstufe definieren. Zudem sollen die Leistungserbringer zur verbindlichen Datenlieferung an diese Organisation und an den Bundesrat verpflichtet werden (Art. 47b KVG neu). Die Vernehmlassung des BAG läuft noch bis zum 14. Dezember 2018.

\section{Monitoring Tarifeingriff des Bundesrates in den TARMED}

Patrick Müller informiert, dass der Bundesrat mit dem zweiten Tarifeingriff den Tarifpartnern auch ein umfangreiches Monitoring mit einer kostenlosen Lieferung an das BAG auferlegt hat. Eine erste Lieferung erfolgte per Juli 2018 über das erste Quartal 2018. Weiter informiert P. Müller, dass die FMH diese erste Lieferung mit einem klaren Disclaimer hinterlegte, da aufgrund der noch unvollständigen Datenstabilität keine Rückschlüsse auf das Abrechnungsverhalten und die Anwendung der Tarifstruktur, insbesondere auch in Bezug auf die neuen Limitationen, hergestellt werden können. Aufgrund der komplexen Umstellung kommt es insbesondere im ersten Quartal zu über-

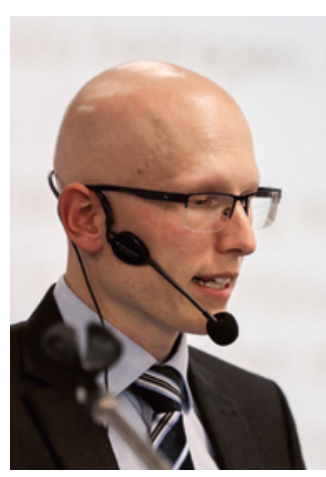

Patrick Müller referiert über die Vernehmlassung des Massnahmenpakets zur Kostendämpfung des Bundesrates und zum Monitoring des zweiten Tarifeingriffes in den TARMED. durchschnittlich vielen Rechnungsstorni, welche sich auch auf die Datenlage auswirken können Je nach technischer Umsetzung können die stornierten Rechnungen nicht aus dem Datensatz entfernt werden und können bei einer grossen Menge an Storni das Gesamtbild verzerren. Verlässlichere Zahlen sind daher erst mit dem Q2 2018 zu erwarten mit einem entsprechenden Delay für den Datenabzug von mindestens 3-4 Monaten. Die FMH hat das BAG zudem in einem Monitoringbericht auf diverse offene Umsetzungsprobleme im Zusammenhang mit den zweiten Tarifeingriff aufmerksam gemacht. Eine weitere Lieferung steht bis Ende 2018 an, bei der erstmals vertiefte Analysen durchgeführt werden können.

\section{Informationen aus dem Laborbereich}

Dr. med. Susanne Christen informiert, dass die Motion 17.3969 «Tarifpartner sollen Tarife von Laboranalysen aushandeln" zunächst vom Ständerat und nun am 19.9.2018 auch vom Nationalrat angenommen wurde und somit die Analysenliste von einem Amtstarif zu 
einem verhandelten Tarif umgewandelt werden soll. Sie verweist zudem auf die Änderungen in der Analysenliste per 1.9.2018. Insbesondere wurde die Nomenklatur des Troponin in der Liste der schnellen Analysen (Position 1734.01) abgeändert; der TropninSchnelltest (Position 1735.00) wurde zwar in der Liste der ergänzenden Analysen gestrichen, ist aber noch

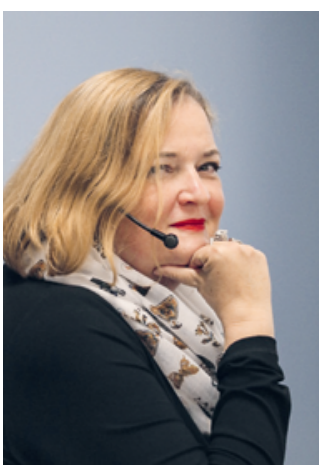

Dr. med. Susanne Christen referiert über die aktuellen Gegebenheiten im Bereich Praxislabor. bei Hausbesuchen zugelassen. Kollegen rechnen zum Teil Analysepositionen aus der erweiterten Liste mit bestimmten Weiterbildungstiteln ab und berufen sich dabei auf die Besitzstandgarantie. Sie erhalten deshalb gerechtfertigterweise Rückforderungen von tarifsuisse: Die Analysenliste ist ein Amtstarif, und dort gibt es keine Möglichkeit eines Besitzstandes; die Besitzstandgarantie betrifft nur den TARMED. Der Fähigkeitsausweis Praxislabor ist ein Bestandteil des Qualitätssicherungsprogramms im Praxislabor. Die Rezertifizierung erfolgt durch die Teilnahme an der externen Qualitätskontrolle. Zur Bestätigung dieser Rezertifizierung hat tarifsuisse begonnen, von einzelnen Ärzten die von den Qualitätskontrollzentren ausgestellten Teilnahmezertifikate der letzten 5 Jahre einzufordern. Gemäss einer Vereinbarung mit santésuisse in der QUALAB ist bei der SASIS AG bei den einzelnen Leistungserbringern hinterlegt, ob sie an den externen Qualitätskontrollen teilnehmen. Dementsprechend empfehlen wir den Ärzten, die Kostenträger wie auch tarifsuisse darauf hinzuweisen, dass sie die Information bei der SASIS AG einholen sollen.

\section{Tarifpartnerschaftliche ambulante Tarifrevision}

Kerstin Schutz, Sabine Zehnder und Christian Oeschger präsentierten den anwesenden Tarifdelegierten die wesentlichen Änderungen in der tarifpartnerschaftlichen Leistungsstruktur. Neben der Aktualisierung der Kostenmodelle ist es auch auf Ebene der Nomenklatur zu Änderungen gekommen. Die Tarifdelegierten der Fachgesellschaften werden über die Veränderungen in ihren Kapiteln durch die Arbeitsgruppenleiter noch ausführlicher informiert und in den kommenden Tagen auch mit Warenkörben bedient. Es ist wichtig, dass die revidierte Leistungsstruktur anhand dieser Zusammenstellungen beurteilt wird und nicht Tarifpositionen 1:1 einander gegenübergestellt werden. Die FMH wird im November 2018 in der $S \ddot{Z} Z$ ausführlich zum Tarif und dem weiteren Vorgehen informieren.

\section{Rückblick und Fazit}

Zum Abschluss fasst Dr. med. Urs Stoffel zusammen, dass der Druck seitens Politik auf die Ärzteschaft weiter zunimmt und die steigenden Gesundheitskosten zum Wahlkampftema 2019 von gewissen Parteien werden. U. Stoffel appelliert an die Anwesenden, dass diese Chance und Möglichkeit genutzt werden soll, um zu beweisen, dass die Ärzteschaft fähig ist, gemeinsam mit den Tarifpartnern einen revidierten, sachgerechten und betriebswirtschaftlichen Tarif zu verhandeln.

Folien: Alle Unterlagen sowie die Gesamtpräsentation finden Sie auf der Website der FMH: www.fmh.ch $\rightarrow$ Ambulante Tarife $\rightarrow$ Tarifdelegierten-Tag $\rightarrow$ 27. September $2018 \rightarrow$ Präsentation.

NächsterTermin: Der nächsteTarifdelegierten-Tag findet am Donnerstag, 11. April 2019, im Hotel Ador in Bern statt. Bitte reservieren Sie sich den Termin!

Korrespondenz:
FMH
Abteilung Ambulante
Versorgung und Tarife
Baslerstrasse 47
CH-4600 Olten
Tel. 0313591230
Fax 0313591238
tarife.ambulant[at]fmh.ch
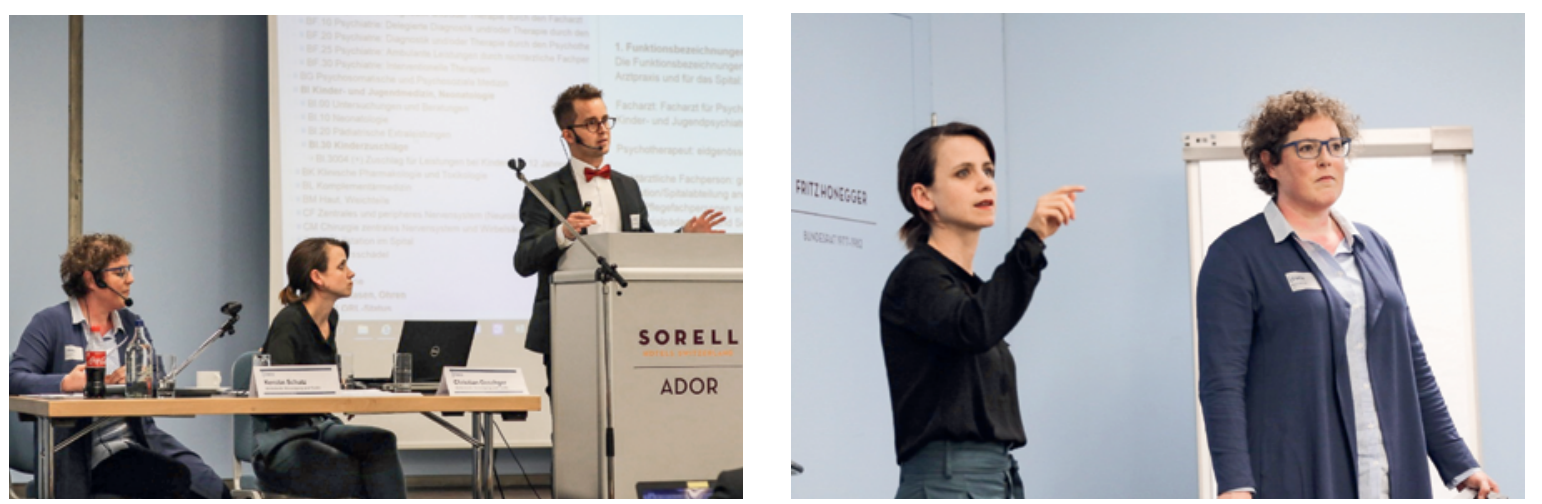

Christian Oeschger, Kerstin Schutz und Sabine Zehnder informieren über die tarifpartnerschaftliche ambulante Tarifrevision. 